\title{
Characterization of the nasopharyngeal microbiota in health and during rhinovirus challenge
}

\author{
E Kaitlynn Allen ${ }^{1,2}$, Alex F Koeppel ${ }^{3}$, J Owen Hendley ${ }^{4}$, Stephen D Turner ${ }^{3}$, Birgit Winther ${ }^{5}$ and Michèle M Sale ${ }^{1,2,6^{*}}$
}

\begin{abstract}
Background: The bacterial communities of the nasopharynx play an important role in upper respiratory tract infections (URTIs). Our study represents the first survey of the nasopharynx during a known, controlled viral challenge. We aimed to gain a better understanding of the composition and dynamics of the nasopharyngeal microbiome during viral infection.
\end{abstract}

Methods: Rhinovirus illnesses were induced by self-inoculation using the finger to nose or eye natural transmission route in ten otherwise healthy young adults. Nasal lavage fluid samples (NLF) samples were collected at specific time points before, during, and following experimental rhinovirus inoculation. Bacterial DNA from each sample ( $N=97$ from 10 subjects) was subjected to 165 rRNA sequencing by amplifying the V1-V2 hypervariable region followed by sequencing using the 454-FLX platform.

Results: This survey of the nasopharyngeal microbiota revealed a highly complex microbial ecosystem. Taxonomic composition varied widely between subjects and between time points of the same subject. We also observed significantly higher diversity in not infected individuals compared to infected individuals. Two genera - Neisseria and Propionibacterium - differed significantly between infected and not infected individuals. Certain phyla, including Firmicutes, Actinobacteria, and Proteobacteria, were detected in all samples.

Conclusions: Our results reveal the complex and diverse nature of the nasopharyngeal microbiota in both healthy and viral-challenged adults. Although some phyla were common to all samples, differences in levels of diversity and selected phyla were detected between infected and uninfected participants. Deeper, species-level metagenomic sequencing in a larger sample is warranted.

Keywords: Microbiota, nasopharynx, longitudinal, sequencing, rhinovirus illness

\section{Background}

The nasopharynx is frequently colonized by both commensal and pathogenic bacteria [1]. Many pathogenic species, including Streptococcus pneumonia, Haemophilus influenza, Moraxella catarrahlis, Staphylococcus aureus, and Neisseria meningitidis, exist in the nasopharynx of apparently healthy individuals [1-4]. Ling et al. found the nasopharyngeal microbiota to be distinct from other

\footnotetext{
* Correspondence: msale@virginia.edu

${ }^{1}$ Center for Public Health Genomics, University of Virginia, PO Box 800717, Charlottesville, USA

${ }^{2}$ Department of Biochemistry and Molecular Genetics, University of Virginia, Charlottesville, USA

Full list of author information is available at the end of the article
}

body sites surveyed (saliva, dominant hands, and feces) in healthy Chinese adults [5].

Understanding the relationships between microbes of the upper respiratory tract (URT) during perturbations is anticipated to provide insights into the pathogenesis of URT infections. Studies of the nasopharyngeal microbiota in children have observed changes due to season (winter/fall versus spring) [4] and treatment with antimicrobials or the heptavalent conjugated pneumococcal polysaccharide vaccine [6,7]. Specific commensal taxa have been negatively associated with colonization of known pathogenic bacteria and with acute otitis media in children, and these relationships changed depending on antibiotic usage [8].

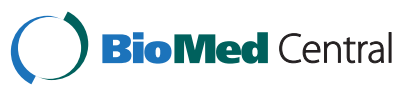


Like pathogenic bacteria, viruses, including rhinoviruses, enteroviruses, coronaviruses, and adenoviruses, have been found in asymptomatic, healthy individuals $[9,10]$. Infections of the URT display complex interactions between bacterial pathogens and viruses, both synergistic and competitive [11-14]. Viruses in the URT can alter bacterial adherence [15], bacterial colonization [16], and host immune response [17-19]. A study of the nasopharyngeal microbiota of children with severe bronchitis showed microbiotal shifts depending on the viral infection (human rhinovirus (HRV) only, respiratory syncytial virus (RSV) only, or co-infection) [20]. The lung microbiota in chronic obstructive pulmonary disease (COPD) patients and healthy individuals during a rhinovirus challenge showed significant changes after infection [21].

We previously used microarray technology to investigate bacteria in the adult nasopharynx prior to, during, and after experimental rhinovirus infection [22]. The microarray approach allowed us to detect bacteria at the species level, but we were only able to test the presence or absence of about 60 clinically informative bacteria. The goal of the present study was to characterize the relative abundance of microbial communities of the nasopharynx at the phylum and genus levels, by sequencing the $16 \mathrm{~S}$ rRNA gene of bacteria in nasal lavage fluid (NLF) samples from the same adults during rhinovirus challenge.

\section{Methods}

\section{Sample collection}

This protocol has been previously described [22]. Briefly, participants were volunteers between the ages of 18 and 65 years, who responded to advertisements in 2010. Subjects were eligible if they had a screening serum neutralizing antibody of 1:4 or less to the challenge Rhinovirus type 39. The study was approved by the Institutional Review Board for Health Sciences Research at the University of Virginia. Informed consent was obtained from all ten participants prior to enrollment. The rhinovirus immunotype 39 inoculum pool has been safety tested and approved for use by the Food and Drug Administration (FDA) (IND12934). This study was conducted with all sample collection during the fall. Subjects were exposed to a total of 100 to 300 TCID50 of rhinovirus in $250 \mu \mathrm{L}$ by self-inoculation by touching the medial cantus and conjunctiva of one eye or the septum in the nasal vestibulum on one side of the nose and repeated once after a 5- to 15-minute period. NLF was obtained by installation of $5 \mathrm{~mL}$ of $0.9 \%$ saline into each nasal cavity which was recovered into a waxed paper cup. The saline is able to reach throughout the nasopharynx due to the position of the participant's head (tilted backwards). Ten nasal washes were obtained from each volunteer: three during the week prior to HRV inoculation, one on each of five days immediately following inoculation, and on days 10 and 21 following inoculation (Figure 1). An aliquot of $1 \mathrm{~mL}$ of each of the NLF sample was placed in tubes containing an equal volume of viral collection broth, kept on ice, and transported to the laboratory within one hour for rhinovirus isolation in tissue cultures. The remaining NLF was transferred to $2-\mathrm{mL}$ cryo-tubes and stored frozen at $-80^{\circ} \mathrm{C}$.

\section{Assessment of infection}

This protocol has previously been described [22]. Briefly, a subject was considered infected if the challenge rhinovirus was detected in the NLF at least once during the five days following inoculation. Additionally, the serum antibody response to the challenge rhinovirus type 39 was examined in sera obtained prior to inoculation and three weeks following inoculation by standard methods [23]. Subjects with a 4-fold increase in antibody titer to the challenge virus were considered to be infected. If either of these tests were positive, the subject was considered infected. Assessment of illness of the participants is described in Additional file 1.

\section{DNA extraction}

DNA was isolated from NLF using bead beating and phenol-chloroform extraction methods. This protocol [24] was optimized from Ren T, et al. to increase DNA output from a more difficult sample type (NLF). Briefly, $1.8 \mathrm{~mL}$ of nasal wash was transferred to a 2-mL BeadBeater tube (BioSpec, Bartlesville, OK) and centrifuged at 2,348 $g$ (5,000 $\mathrm{rpm})$ for 10 minutes. Supernatant $(1.3 \mathrm{~mL})$ was aspirated, leaving all pelleted bacterial cells plus $500 \mu \mathrm{L}$ supernatant for DNA extraction and subsequent sequencing. Then, 20 $\mu \mathrm{L}$ Proteinase K (20 mg/mL) (Roche, Basel, Switzerland)

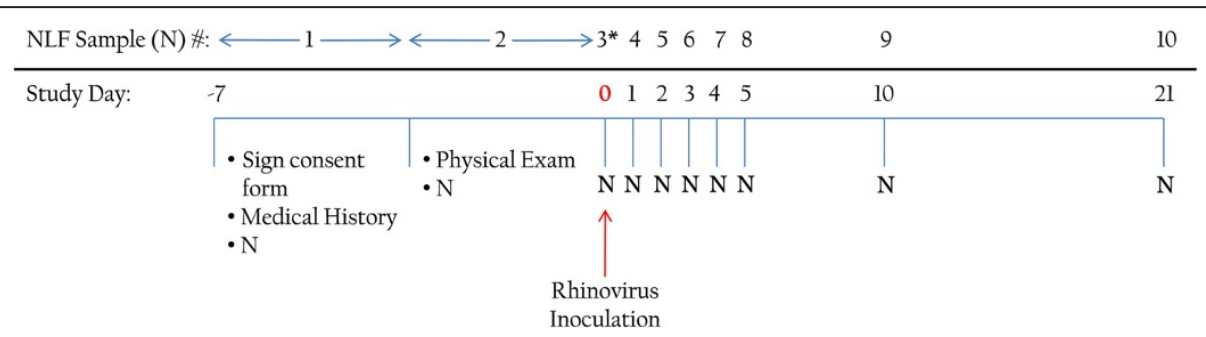

Figure 1 Study timeline including when information or samples were collected from study participants. *Day on which nasal lavage fluid (NLF) samples were obtained prior to rhinovirus inoculation. 
was added and incubated at $60^{\circ} \mathrm{C}$ for two hours then $95^{\circ} \mathrm{C}$ for 10 minutes. A total of $200 \mu \mathrm{L} 10 \%$ SDS (Fischer Scientific, Waltham, MA), $400 \mu \mathrm{L}$ of $0.1 \mathrm{~mm}$ zirconia/silica beads (BioSpec, Bartlesville, OK), $100 \mu \mathrm{L}$ of buffer $(10 \mathrm{mM}$ Tris- $\mathrm{HCl} \mathrm{pH}$ 7.5, $5 \mathrm{mM}$ EDTA, $100 \mathrm{mM} \mathrm{NaCl}$ ), and 500 $\mu \mathrm{L}$ of phenol:chloroform:isoamyl alcohol (25:24:1) (Fischer Scientific, Waltham, MA) were added to the tube. The sample was homogenized on a Biospec Mini-BeadBeater-8 for 3 minutes and then centrifuged for 10 minutes at $10,000 \mathrm{~g}$. The top aqueous layer was transferred to a new Phase-Lock-Gel tube (VWR, Radnor, PA) with an equal volume of chloroform:isoamyl alcohol (24:1) (Acros Organics, Fischer Scientific, Waltham, MA) and centrifuged at 20,000 $g$ for 10 minutes. This step was repeated twice to ensure removal of phenol. The sample was then ethanol precipitated, and the DNA pellet was resuspended in $50 \mu \mathrm{L} 10$ $\mathrm{mM}$ Tris- $\mathrm{HCl} \mathrm{pH}$ 8.0. Each set of extractions was accompanied by a negative control to ensure no contaminants in the extracted DNA.

\section{Tag-PCR amplification of the $\mathrm{V} 1-\mathrm{V} 2$ regions of the bacterial 16S rRNA gene and pyrosequencing}

This protocol has been previously described [24]. Briefly, the V1-V2 hypervariable regions of the $16 \mathrm{~S}$ rRNA gene were amplified from extracted DNA samples using two primers containing the universal sequences $27 \mathrm{~F}$ (5'-AG RGTTTGATCMTGGCTCAG-3') and 534R (5'-TTACC GCGGCTGCTGGCAC-3'). Each sample was tagged with a unique barcode $(10 \mathrm{bp})$ added to the $5^{\prime}$ end of the forward primer. Conditions for amplification were $94^{\circ} \mathrm{C}$ for 3 minutes, then 30 cycles of $94^{\circ} \mathrm{C}$ for 30 seconds, $57^{\circ} \mathrm{C}$ for 45 seconds, and $72^{\circ} \mathrm{C}$ for 60 seconds with a final extension step of $72^{\circ} \mathrm{C}$ for 5 minutes. Each set of samples was amplified with a negative control to ensure no contaminants from reagents. PCR products were then run on a gel to verify amplification of the correct size. Amplicons from all samples were quantified using the Qubit ${ }^{\bullet}$ 2.0 Fluorometer and then pooled in equal molar ratios with 49 samples in pool 1 and 48 samples in pool 2. To achieve a final pool of $20 \mu \mathrm{L}$ of sample with a $10 \mathrm{ng} / \mu \mathrm{L}$ concentration, $40.8 \mathrm{ng}$ of each sample were added to pool 1 and $41.67 \mathrm{ng}$ were added from each sample to pool 2. The pooled samples were then gel-purified and the concentration of each sample was measured using the Qubit 2.0 Fluorometer to ensure the minimum of $20 \mu \mathrm{L}$ of $10 \mathrm{ng} / \mu \mathrm{L}$ sample for sequencing. Each pooled sample was sequenced using titanium chemistry on a 454 Life Science Genome Sequencer FLX platform at the University of Virginia Department of Biology Genome Core Facility using the standard protocol for sequencing. The samples were run on an Agilent 2100 Bioanalyzer HighSensitivity chip to determine the size distribution of the library samples. Then, a KAPA Biosystems qPCR assay was run to determine the effective concentration of the samples.

\section{Quality assessment and filtering of sequences}

This quality of the read sequences was assessed using FastQC v0.10.1 [25]. This assessment revealed that a significant fraction of our reads were identical short $(<100$ bp) reads (homopolymers). Based on this assessment, and the distribution of read lengths, we filtered the sequences by length, removing any reads shorter than 100 bp in length, or greater than $600 \mathrm{bp}$. This filter alone reduced our initial dataset from 4.32 $\mathrm{Mbp}$, to $3.32 \mathrm{Mbp}$ (approximately $23.1 \%$ reduction). In addition, reads with an average quality score lower than 25 (phred scale) were removed, as were any reads that contained 50-base windows with average quality scores below that threshold. Qiime's default filters also removed any reads with mismatches in the primer or barcode sequences, and any reads with more than six ambiguous bases.

\section{Clustering and filtering of operational taxonomic units (OTUs)}

Sequences were clustered into operational taxonomic units (OTUs) (97\% identity cutoff) using the uclust algorithm v1.2.22 (de novo clustering) [26] in QIIME v1.8 [27], and assigned taxonomy using RDP classifier v2.2 [9], trained with the Greengenes (gg_13_8) [28] dataset. OTUs were removed from the dataset if they could not be classified at the domain level, or were classified as archaea, or were classified as chloroplast sequences. OTUs estimated to be putatively chimeric by the uchime algorithm v4.2.40 [29] were also filtered out, as were singleton OTUs. After filtering, we were left with a mean of 1021.6 sequences per sample (SD 630.84, median 913, minimum 134, maximum 4,501). Samples containing fewer sequences than three SD below the mean count (146 sequences/sample) $(n=1)$ were eliminated from downstream analyses.

\section{Sequence analysis}

Analyses of taxonomic composition and diversity were performed using QIIME, version 1.8 [27]. Default parameters were used for the core analyses. OTU representatives were aligned with pyNAST v1.2.2 [30], and phylogenies were generated using FastTree v2.1.3 [31]. Alpha diversity (within-population diversity) of samples was measured using counts of observed species (OTUs), the chao1 estimator for species richness, and the Shannon diversity index, which estimates total diversity taking into account both species richness and evenness. Beta diversity (diversity between populations) was calculated using UniFrac distances (unweighted) [32] between samples (based on the relative abundance of OTUs), and visualized using principal coordinates analysis (PCoA) [33]. In order to compare diversity of OTUs in the nasopharynx to that of other body sites, we also performed a combined beta diversity analysis of our nasopharyngeal samples with samples from several different body sites (gut, oral cavity, external auditory canal, 
nostril, and hair) from Costello et al. [34], as well as adenoid samples from Ren et al. [24].

\section{Statistical analysis}

Analyses of phylum abundance between inoculation states (before, during, and after) and between subjects, was performed using analysis of variance (ANOVA). For significant results, the Tukey Kramer test was subsequently conducted. ANOVA was also used for the analyses of class, order, and family abundances between inoculation states (before, during, and after). Comparisons of alpha diversity between the infected and noninfected sample groups were done using Mann-Whitney Wilcoxon tests, as were the abundances of selected genera between the infected and non-infected samples, and the comparison of inter-individual and intra-individual Unifrac distances.

\section{Results}

\section{Subject population}

Ten volunteers were eligible and enrolled into the study. Forty percent of volunteers were female, and the average age of volunteers was 19.6 years. Six of the subjects were self-inoculated into the nose and four were selfinoculated into the eye. Seven out of the ten subjects were infected by the rhinovirus challenge. Out of the infected subjects, three were considered ill based on the modified Jackson cold method (Additional file 1). All subjects were sampled at all ten time points. Demographic information and information on infection status is included in Table 1.

DNA was successfully extracted from 99/100 NLF samples with concentration values ranging from $5.86 \mathrm{ng} / \mu \mathrm{L}$ to $33.8 \mathrm{ng} / \mu \mathrm{L}$, which is above the recommended threshold for microbiota studies [35]. One sample (subject number 6, day 3) did not have enough NLF for DNA extraction. PCR amplification and pyrosequencing of the V1-V2 hypervariable regions of the bacterial 16S rRNA gene was successful in 97 out of the 99 NLF samples. PCR amplification was unsuccessful in two samples (subject number 3, day 2 and subject number 10, day 3). Sequencing of the two pools of DNA extracted from the NLF samples resulted in 679,135 reads. These reads were then quality filtered to select the most reliable reads for analysis (see Methods). After all quality filters had been performed on both raw sequences and OTUs (see Methods), there were 99,095 reads remaining for analysis (median of 913 reads per sample) (Figure 2). After filtering, reads had a mean length of 376 bp (median $414 \mathrm{bp}$ ), and one sample (subject number 10, day 7 (134 reads)) was excluded for containing fewer sequences than $3 \mathrm{SD}$ below the mean count (146 sequences/ sample).

\section{Analysis of nasopharyngeal bacterial richness and composition by sequencing}

The sequences were clustered into 3,229 distinct OTUs (97\% identity cutoff). Singleton OTUs were filtered out, leaving 1,608 OTUs for downstream analysis. Across all ten subjects, the dominant phyla were Firmicutes (40.6\%), Actinobacteria (20.9\%), Proteobacteria (17.1\%), and unclassified (20.6\%), with the relative abundance of each phylum being highly variable across the subjects (Additional files 2 and 3 ) and time points (Additional files 2 and 4). To determine if bacterial composition changes occur due to viral infection, we compared the relative abundance of the dominant phyla before (time points 1 to 3 ), during (time points 4 to 8 ), and after (time points 9 to 10) Rhinovirus infection in infected and not infection subjects (Figure 3). No phylum showed a significant change in relative abundance between time points. Additionally, we performed ANOVA on the relative abundances (log-transformed values), which revealed no significant differences (after FDR correction) in the abundance of the three dominant phyla before, during, or after inoculation, in either the infected or uninfected categories. Significant differences were found in two subjects: subject number 5 , in which Proteobacteria abundance was significantly different before inoculation than during or after inoculation $(P=0.039)$, and subject number 9, in which Actinobacteria abundance was

Table 1 Subject information

\begin{tabular}{lllllllllll}
\hline & \multicolumn{7}{c}{ Subject number } \\
\cline { 2 - 9 } Variable & $\mathbf{1}$ & $\mathbf{2}$ & $\mathbf{3}$ & $\mathbf{4}$ & $\mathbf{5}$ & $\mathbf{6}$ & $\mathbf{7}$ & $\mathbf{8}$ & $\mathbf{9}$ & $\mathbf{1 0}$ \\
\hline Age, years & 19 & 19 & 18 & 18 & 20 & 21 & 21 & 20 & 19 & M \\
Sex & $\mathrm{F}$ & $\mathrm{F}$ & $\mathrm{F}$ & $\mathrm{M}$ & $\mathrm{F}$ & $\mathrm{M}$ & $\mathrm{M}$ & $\mathrm{M}$ & $\mathrm{M}$ & $\mathrm{M}$ \\
Race & White & White & White & White & White, Asian & White & White & White & African American & White \\
Route of inoculation & Eye & Nose & Nose & Nose & Nose & Eye & Eye & Eye & Nose & Nose \\
HRV infection & yes & no & yes & yes & yes & no & yes & yes & no & yes \\
Criteria for infection status & Ab & - & HRV/Ab & HRV & HRV/Ab & - & HRV/Ab & Ab & - &
\end{tabular}

'The subject did not specify "Non-hispanic." For criteria for infection status, HRV indicates HRV shedding in at least one sample during time points 4 to 8 (during the infection period) and $A b$ indicates that the subject had a 4 -fold rise in serum-neutralizing antibody in response to the challenge virus. $M$, male; $F$, female. 


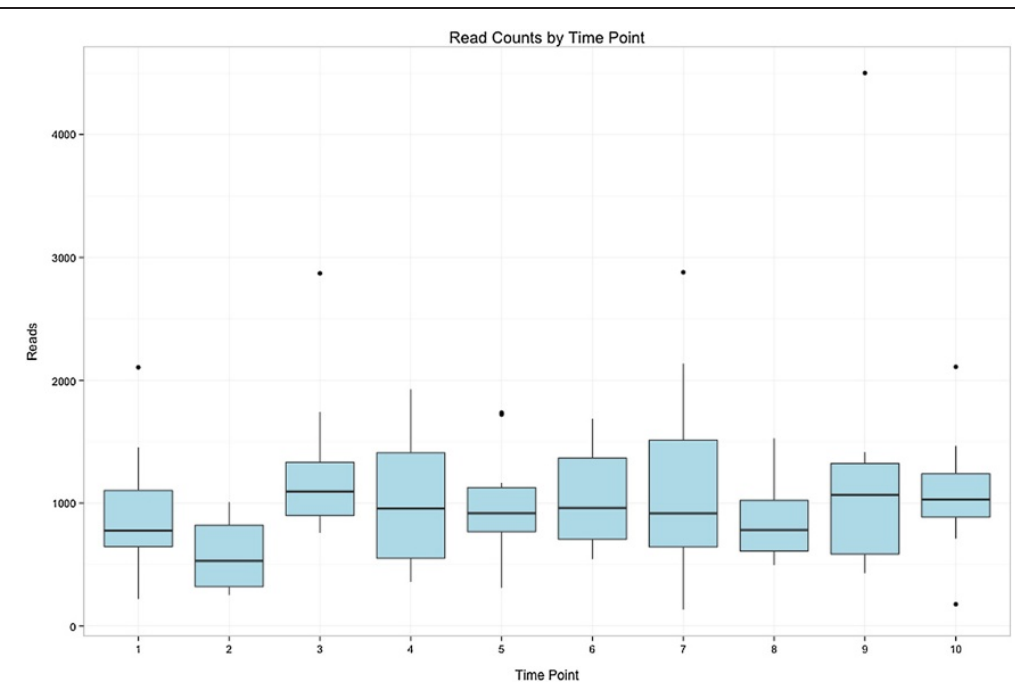

Figure 2 Box and whisker plot of read counts at each time point during the study after quality control filtering.

significantly different between the before, during, and after time points $(P=0.0001)$ (Additional file 5$)$. No significant differences were found in the abundances of dominant classes, orders, or families before, during, or after inoculation, in either infected or not infected individuals (all $P$-values $>0.11$ ). ANOVA was also performed to test the null hypothesis that the relative abundances (log-transformed values) of the dominant phyla were the same between subjects (Additional file 6). After FDR correction, there was a significant difference in the relative abundance of Firmicutes between subjects
$\left(P_{\text {adj }}=0.009\right.$ in not infected subjects, $P_{\text {adj }}=0.001$ in infected subjects).

We also carried out a rarefaction analysis to compare bacterial species richness between the samples before, during, and after infection by plotting the rarefaction measure (the Chao1 diversity index for OTUs at 97\% identity threshold) by the number of sequences per sample (Figure 4; Additional files 7 and 8). The rarefaction plot curves indicate that the nasopharynx, sampled by NLF, is a very complex microbial environment. Deeper sequencing could reveal even more diversity in this very

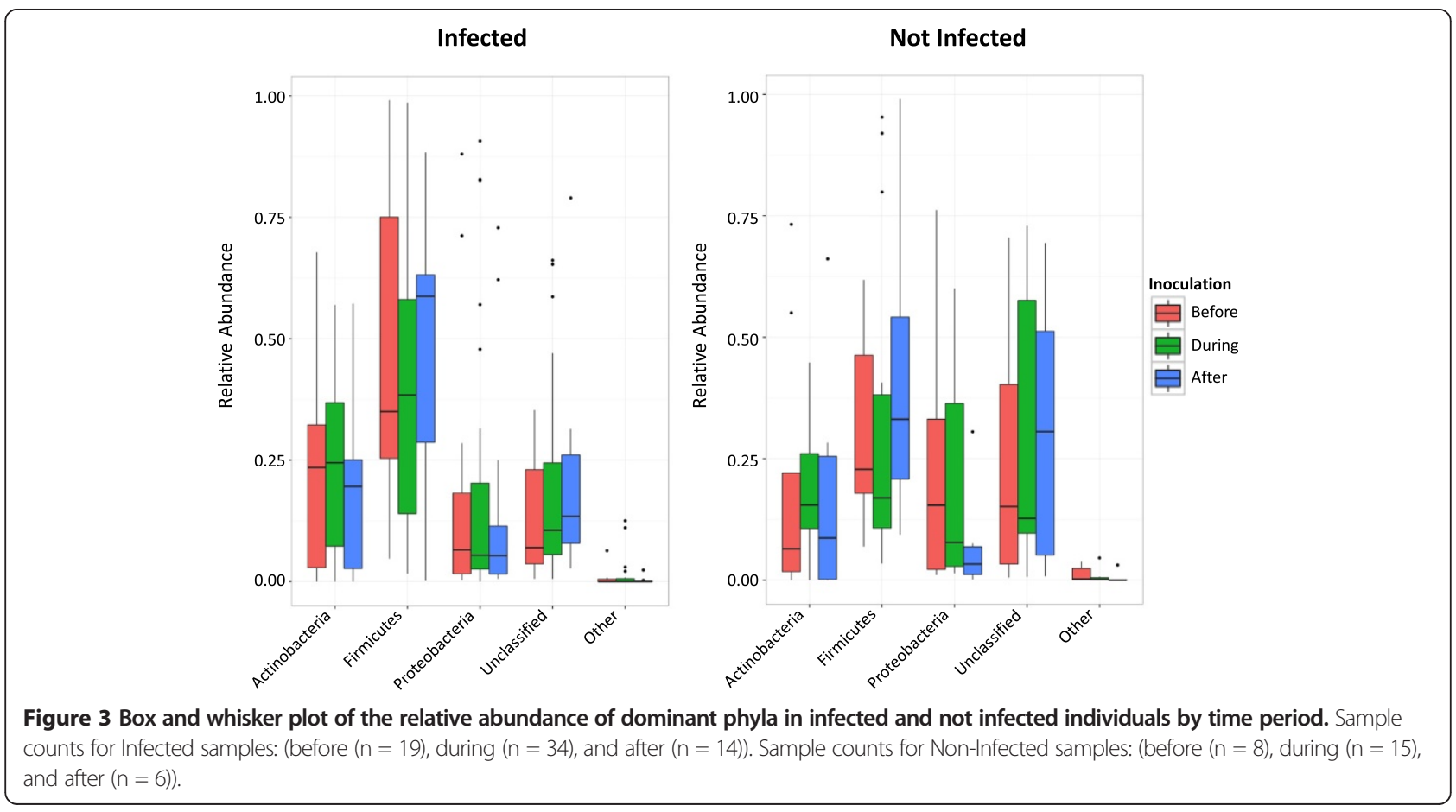




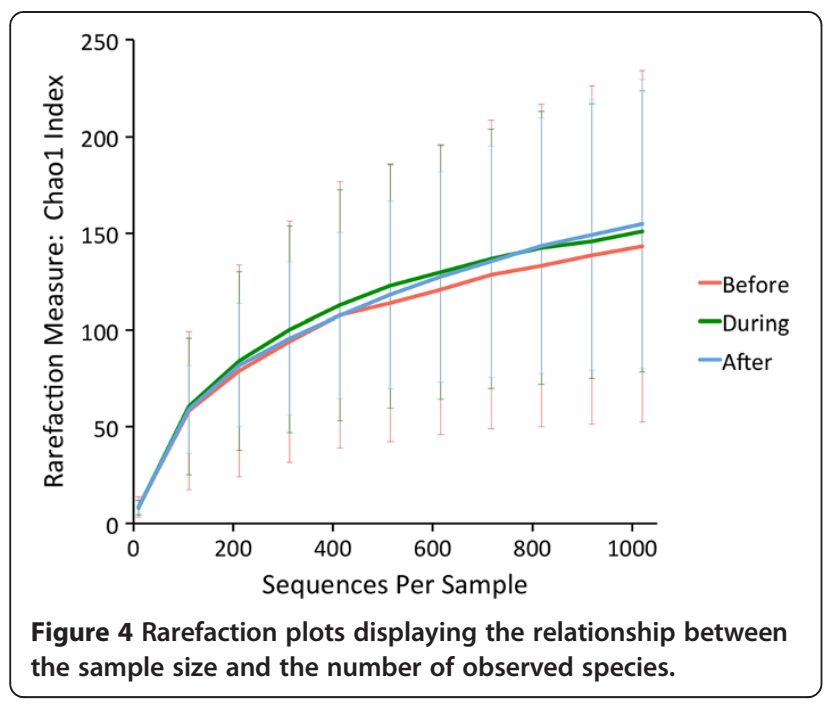

complex microbiota. Additionally, there is no significant difference between the species richness between the three time points, though there is a trend for more bacterial richness in the time points during and after inoculation than in the time points before inoculation.

The most abundant genus in the nasopharyngeal microbiota across samples was Alloiococcus $(21.2 \%$ of total abundance), followed by Corynebacterium (14.7\%), Staphylococcus (8.9\%), Haemophilus (5.3\%), Propionibacterium (4.6\%), and Streptococcus (3.2\%). All other classified OTUs belonged to genera comprising less than $2 \%$ of the total abundance. It should be noted that these abundances reflect total abundance across all samples. Taxonomic composition varied widely between samples (Additional file 2).

We compared the microbiota of the nasopharynx (NLF samples) to those of other body sites including the gut, oral cavity, external auditory canal, nostril, hair, and adenoid using PCoA (Figure 5; Additional files 9 and 10). For the purpose of comparison, only the NLF samples taken prior to inoculation were included for this analysis. The microbiota of the nasopharynx is most similar to the external auditory canal, nostril, hair, and adenoid. The gut and oral cavity are distinct from the rest of the samples. When we used PCoA to compare the NLF samples before, during, and after infection; infected versus not infected; ill versus not ill; and by route of infection, we saw no distinct differences in the samples (Additional files 11 and 12). When looking at the PCoA to compare NLF samples between each subject (Additional file 11), there was no distinct clustering by subject. When comparing NLF samples within each subject (Additional file 12), there was also no distinct clustering. Unifrac distances between samples from different individuals was significantly greater than the distances between samples from the same individual (Figure 6A). Inter-individual Unifrac distances were not significantly different between infected and not infected subjects (Figure 6B).

\section{Bacterial community structure of infected and not infected subjects}

The community structure of bacteria in the NLF samples of infected $(n=7)$ and not infected $(n=3)$ subjects were compared at the genus level. Both groups showed similar

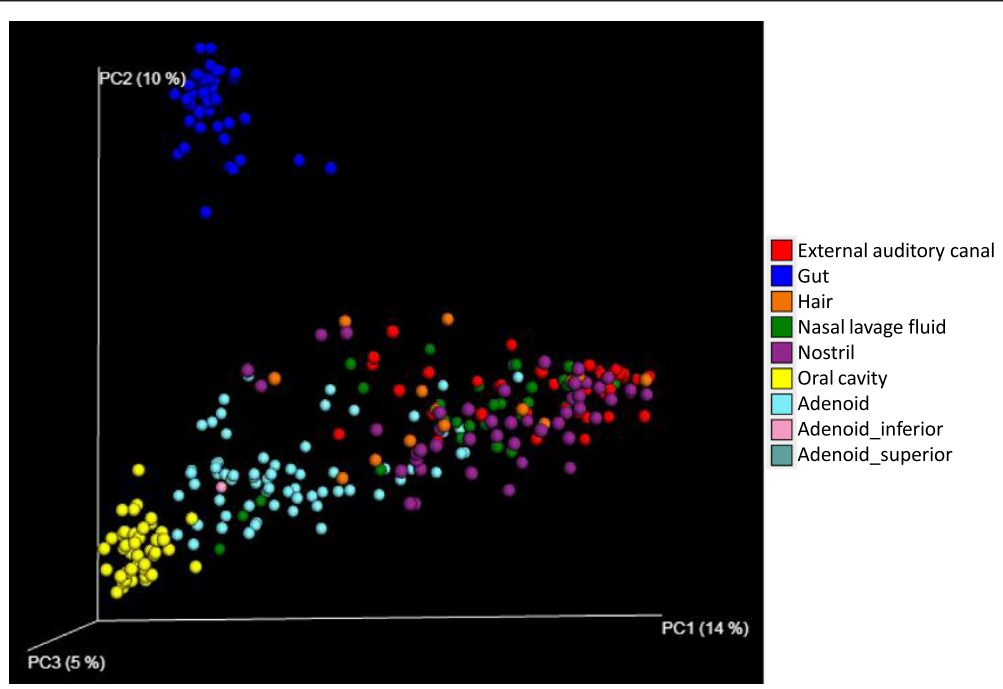

Figure $\mathbf{5}$ Nasopharyngeal microbiota is similar to neighboring body parts, but distinct from gut and oral cavity. Principal coordinates analysis (PCOA) was performed using the unweighted UniFrac distance matrix using nasal lavage fluid (NLF) samples from pre-inoculation time points. Each sample is represented by a point with NLF $(n=27)$ in green, gut $(n=45)$ in dark blue, oral cavity $(n=46)$ in yellow, external auditory canal $(n=44)$ in red, nostril $(n=46)$ in purple, hair $(n=14)$ in orange, and adenoids $(n=69)$ in light blue. 
A

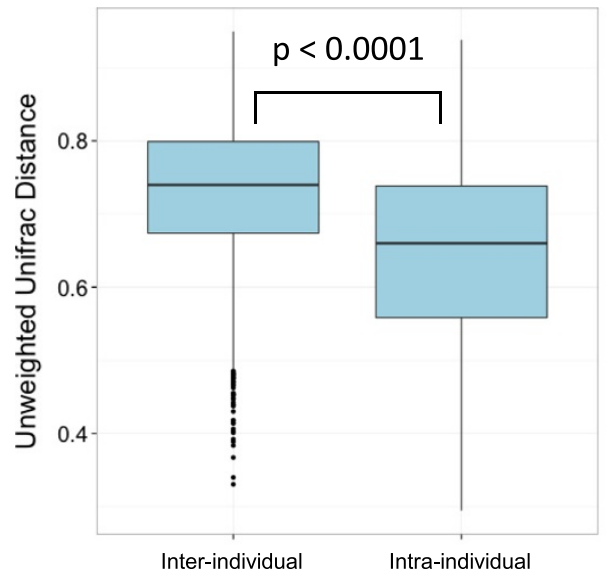

B

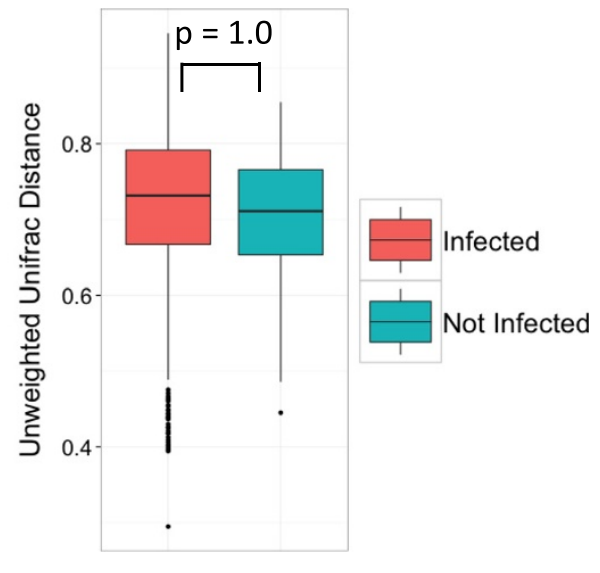

Figure 6 Unifrac distance comparisons. (A) Unweighted Unifrac distances between samples from different individuals (inter-individual) and the distances between samples from the same individual (intra-individual). (B) Inter-individual unweighted Unifrac distances compared between infected and not infected subjects. P-values displayed are the results of Mann-Whitney Wilcoxon tests.

patterns of genera distribution (Figure 7A), but there were significant differences $(P=0.031)$ in the overall community diversity (Chao1 index) between infected and not infected subjects (Figure 7B). The Mann-Whitney Wilcoxon test was used to compare the relative abundances between infected and not infected samples of all genera comprising $1 \%$ or more of the total abundance across samples. Significant differences were detected in two genera (Neisseria and Propionibacterium) after using the Benjamini and Hochberg false discovery rate correction for multiple comparisons (Table 2). Prior to correction for multiple comparisons, the genus Haemophilus was significantly less abundant in the infected samples, suggesting a potential association that could be further investigated with additional samples.

\section{Discussion}

This study is the first to survey and describe the microbiota of the nasopharynx in healthy adults during a rhinovirus challenge. We found a highly complex microbiome that varied between individuals, and significant differences in two genera (Neisseria and Propionibacterium) between infected and not infected individuals. Strengths of our study include a controlled rhinovirus study, longitudinal analyses, and use of highthroughput sequencing technology. Limitations include the modest sample size and sequencing depth.

The results from this survey have been compared to the results of the microarray study [22] using the same NLF samples. Due to the design of the microarray study, it can only detect the presence or absence of bacterial species on the panel included, while the $16 \mathrm{~S}$ method is able to determine relative abundance of all bacteria in the sample. We can only compare the number of mismatches in those genera that the microarray was able to detect $(n=8)$. Out of the total 96 samples, the percent of samples which were detected in one study but not the other ranged from $4 \%$ to $53 \%$ in each genus. These results can be due to two aliquots from the same NLF sample extracted using two different methods. It could also be due to the microarray detecting species in very low abundance because of enrichment due to PCR.

Due to the level of complexity of the nasopharyngeal microbiota, it is possible that significant changes in the bacterial community before, during, and after infection could likely be detected with deeper sequencing and/or recruiting more subjects. Some large changes in relative abundances of phyla within individuals at close time points were observed. It will be important to investigate whether these reflect true biological fluctuations in this dynamic ecological niche or low microbial density via greater sequencing depth. Additionally, using true shotgun metagenomics and taxonomic classification [36] to distinguish bacteria at the species level may allow significant changes in pathogenic and opportunistic bacteria during infection to be discovered. Information on the species level is especially important because, in numerous cases, there are pathogenic and commensal bacteria within the same genus, for example, Haemophilius influenzae, a known pathogen, and its closest phylogenetic relative Haemophilus haemolyticus, a commensal bacterium $[37,38]$.

PCoA of the nasopharyngeal microbiota samples along with samples from various other body habitats shows logical clustering with similar locations throughout the URT. The nasopharynx, adenoid, ear, hair, and nostril samples all formed a single large cluster. While this cluster was distinct from the separate clusters formed by the 


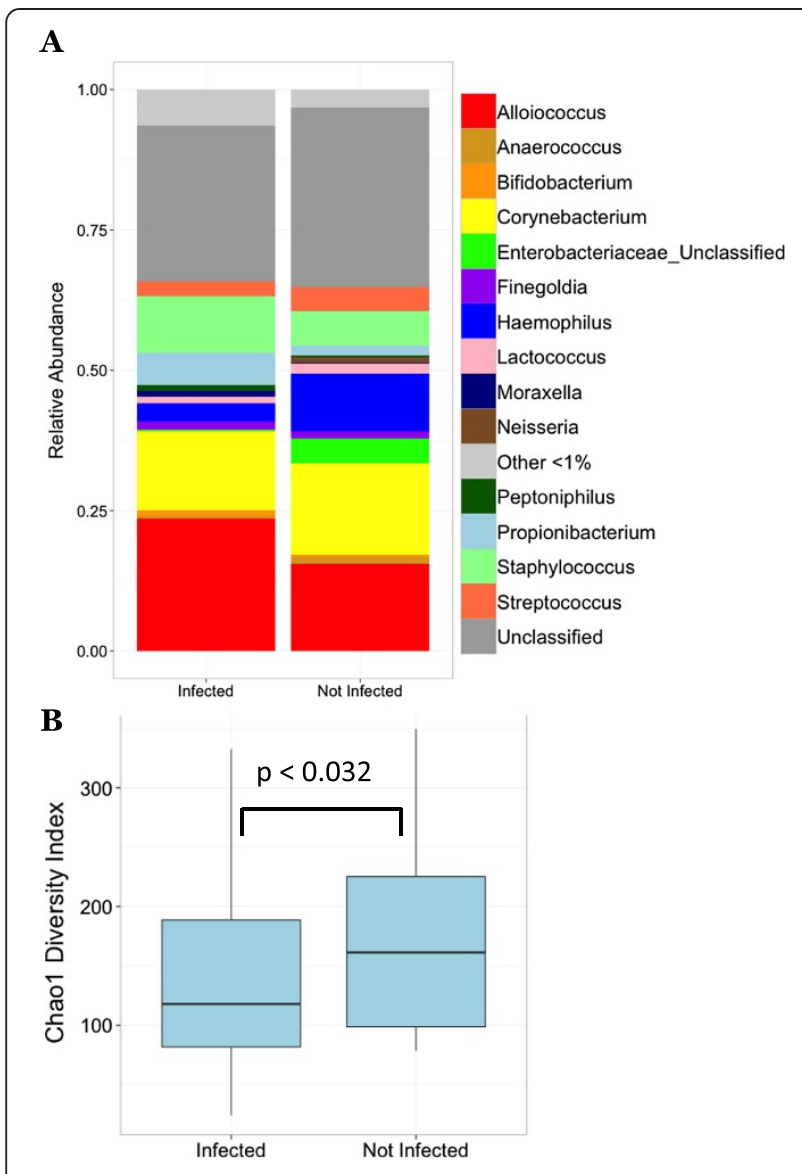

Figure 7 Stacked taxonomic bar chart (A) with box and whisker plot (B) - Shannon diversity index (Not infected $(n=29)$ versus Infected $(\mathbf{n}=\mathbf{6 7})$. Operational taxonomic units (OTUs) were categorized as "Unclassified", if RDP classifier could not classify them to at least the family level. Genera that could be classified to the family level, but not the genus level were categorized by the family name, followed by "_Unclassified" to indicate that the genus is not known. All genera comprising less than $1 \%$ of the total abundance (whether classified or not) were combined into the "Other $<1 \%$ " category.

gut and oral samples, there was not a clear differentiation between the other body sites. Bacterial niches in close proximity have some sharing of bacteria due to physiological events like sneezing and nose blowing [39-41], which could help to explain the overlap between the microbiota of the nasopharynx and other proximal body habitats. Differences in age and health status of subjects along with season of sample collection may impact results. Participants in the Costello, et al. study included healthy adults, with two samples collected during June and two during the following September [34]. Adenoids in the Ren, et al. study were from children undergoing adenoidectomy, collected year round [24]. Demographic data was not accessible because these were discarded surgical specimens. NLF samples in our study were collected during the fall season in healthy adults. Future studies will need to sample complementary niches in the URT to understand coordinated changes of bacterial communities in response to viral infection.

In our study, we surveyed the microbiota of the healthy nasopharynx during viral infection and cataloged a highly diverse population of microbes. The nine dominant phyla of the adenoid microbiota [24] contained the dominant phyla found in our study including Firmicutes, Proteobacteria, and Actinobacteria. We found the same dominant phyla as a study of nasopharyngeal microbiota of healthy Chinese adults [5]. We did not observe an effect of viral infection on microbial composition, which is likely due to a small population, insufficient sequencing depth to detect the full complexity of the microbiota, and the inability to classify OTUs to the species level. In addition to deeper sequencing to achieve species level information, shotgun metatranscriptomics could be used as a complementary approach, to characterize the changes in gene expression that the invasion of a virus can elicit in a microbial community [42]. This information would help us to address the open question of the functional role that the microbiota plays in viral infections. It is uncertain, for instance, whether the microbiota can help to protect the host from viral infection by providing a barrier or by initiating an immune response. Alternatively, it is also possible that the microbiota can aid a virus in invading the host mucosa. One metagenomic study of the airway DNA virome of cystic fibrosis (CF) and non-CF individuals showed that the metabolic profiles of CF and nonCF individuals were distinctly different [43]. This study used the approach of finding target pathways that have altered expression levels in the diseased state, rather than taxonomic composition differences of the microbiota to discover novel therapeutic strategies. Our study has highlighted the highly diverse microbial composition of the adult nasopharynx in both healthy individuals and during viral infection.

Our finding that each individual had a unique nasopharyngeal microbiota broadened our views on how to study and treat URTIs. Most prior studies have looked at a population of individuals with a specific URTI or complication (that is, sinusitis) and sampled each individual for specific pathogens. From the findings of such studies, conclusions may be drawn as to which pathogens are likely involved and targeted treatments may be created. However, with a new understanding of the complexity of the NP microbiota at the individual level, we can apply this understanding by recognizing that future studies need to sample patients with URTIs or complications at baseline comprehensively, and then during acute disease to determine which bacteria respond, expand, disappear. This approach is anticipated to lead to 
Table 2 Comparisons of genus abundance between infected and uninfected samples, using the Mann-Whintey Wilcoxon test

\begin{tabular}{|c|c|c|c|c|c|}
\hline \multirow[b]{2}{*}{ Genus } & \multicolumn{3}{|c|}{ Mean Relative Abundance } & \multirow[b]{2}{*}{$P$-value } & \multirow[b]{2}{*}{$P$-adj } \\
\hline & Infected & Not infected & w & & \\
\hline Alloiococcus & 0.237 & 0.155 & 1198 & 0.071 & 0.227 \\
\hline Anaerococcus & 0.004 & 0.013 & 801 & 0.137 & 0.274 \\
\hline Bifidobacterium & 0.010 & 0.004 & 975.5 & 0.969 & 0.970 \\
\hline Corynebacterium & 0.141 & 0.162 & 946 & 0.842 & 0.970 \\
\hline Enterobacteriaceae_unclassified & 0.003 & 0.043 & 864 & 0.343 & 0.534 \\
\hline Finegoldia & 0.015 & 0.013 & 1166 & 0.113 & 0.263 \\
\hline Haemophilus & 0.032 & 0.104 & 709 & 0.020 & 0.095 \\
\hline Lactococcus & 0.013 & 0.016 & 898.5 & 0.227 & 0.398 \\
\hline Moraxella & 0.009 & 0.002 & 976 & 0.970 & 0.970 \\
\hline Neisseria & 0.001 & 0.011 & 667 & 0.001 & 0.020 \\
\hline Peptoniphilus & 0.009 & 0.003 & 1176.5 & 0.081 & 0.227 \\
\hline Propionibacterium & 0.058 & 0.018 & 1315.5 & 0.006 & 0.042 \\
\hline Staphylococcus & 0.101 & 0.061 & 964.5 & 0.959 & 0.970 \\
\hline Streptococcaceae_unclassified & 0.027 & 0.043 & 871.5 & 0.417 & 0.583 \\
\hline
\end{tabular}

$P$-values $<=0.05$ (with and without false discovery rate correction) are bolded.

improved understanding of disease and more tailored approaches to treatments of these diseases.

\section{Conclusions}

In conclusion, we have conducted a longitudinal survey of the nasopharyngeal microbiota of healthy adults during rhinovirus challenge. This microbiota is highly diverse and varies greatly between individuals. The microbiota of each individual differs between time points throughout the viral challenge, though variability within individuals was less than between individuals. No significant changes in bacterial presence or relative abundance between time points were found due to viral infection. Further studies are needed to further characterize this microbiota and the response of the microbiota to viral infection down to the species level using deeper sequencing. Additionally, metagenomic sequencing will give us insight into transcriptional changes of the host and microbiota during viral infection. With this knowledge, we will better understand the pathogenesis of URT infections.

\section{Availability of supporting data}

The additional files and data set supporting the results of this article are available in Figshare, http://dx.doi.org/ 10.6084/m9.figshare.832471.

\section{Additional files}

Additional file 1: Word document. Assessment of illness. This document describes how subjects were assessed for clinical illness. This information was only used for Beta diversity analyses (IIIness; Additional files 11 and 12).

Additional file 2: Qiime taxonomic composition results (compressed). Taxa summary plots for all samples. Bar and area charts describing the taxonomic composition of all samples analyzed at all taxonomic ranks from genus to phylum.

Additional file 3: Qiime taxonomic composition results (compressed). Taxa summary plots organized by subject. Bar and area charts describing the taxonomic composition of all samples analyzed at all taxonomic ranks from genus to phylum, organized by subject.

Additional file 4: Qiime taxonomic composition results (compressed). Taxa summary plots organized by time point. Bar and area charts describing the taxonomic composition of all samples analyzed at all taxonomic ranks from genus to phylum, organized by time point.

Additional file 5: Excel file. Analysis of variance (ANOVA) test of dominant phyla in each of the subjects. Results for ANOVA test on the relative abundances (log-transformed values) of the three dominant phyla before, during, or after inoculation, in either the infected or uninfected categories.

Additional file 6: Excel file. Analysis of variance (ANOVA) test of dominant phyla between subjects. ANOVA to test the null hypothesis that the relative abundances (log transformed values) of the dominant phyla were the same between subjects in infected and not infected groups.

Additional file 7: Compressed file. Alpha diversity_150. Alpha diversity analyses including rarefaction plots. Plots go to minimum sequences/ sample (approximately 150).

Additional file 8: Compressed file. Alpha diversity_1000. Alpha diversity analyses including rarefaction plots. Plots exclude samples with fewer than the mean sequences/sample (approximately 1000).

Additional file 9: Compressed file. Beta Diversity_All body sites_ pre-inoculation nasal lavage fluid (NLF). Beta diversity (both weighted and unweighted Unifrac) analyses/emperor plots for all body sites, including pre-inoculation nasal wash samples.

Additional file 10: Compressed file. Beta Diversity_All body sites_all nasal lavage fluid (NLF). Beta diversity (both weighted and unweighted 
Unifrac) analyses/emperor plots for all body sites, including all nasal wash samples.

Additional file 11: Compressed file. Beta Diversity_ nasal lavage fluid (NLF) samples only. Beta diversity (both weighted and unweighted Unifrac) analyses/emperor plots for the nasal wash data only.

Additional file 12: Compressed file. Beta Diversity_ nasal lavage fluid (NLF) samples only_by subject. Beta diversity (both weighted and unweighted Unifrac) analyses/emperor plots for the nasal wash data only, split up by subject.

\section{Abbreviations}

ANOVA: analysis of variance; Bp: base pairs; CF: cystic fibrosis; COPD: chronic obstructive pulmonary disease; NLF: nasal lavage fluid - the sample type used to comprehensively survey the microbiota of the nasopharynx;

OTU: operational taxonomic unit - approximations of bacterial species based on a percent similarity threshold for DNA sequence identity at a specified locus (usually 165 rRNA). PCoA: principal coordinates analysis - method used to visualize beta diversity between samples using UniFrac distances; URT: upper respiratory tract - URT refers to the parts of the respiratory tract including the nasal cavity the pharynx, and the larynx; URTI: upper respiratory tract infection - general term used to refer to acute infections of the URT which are usually caused by viral infection.

\section{Competing interests}

The authors declare that they have no competing interests.

\section{Authors' contributions}

EKA carried out molecular genetic studies (DNA extraction, PCR amplification, sequencing preparation), participated in data analysis, and drafted the manuscript. AK carried out data analysis, carried out statistical analyses, and helped in drafting the manuscript. JOH participated in study design and sample recruitment. SDT participated in data analysis and statistical analyses. BW conceived of the viral challenge study, coordinated study recruitment, and carried out sample processing and storage. MMS conceived of the microbiota study and coordinated molecular genetic studies. All authors read and approved the final manuscript.

\section{Acknowledgements}

We thank Kathleen Ashe for her technical assistance throughout this study. The rhinovirus inoculum pool was kindly provided by RB Turner. This study was supported in part by the Pendleton Pediatric Infectious Disease Laboratory at the University of Virginia (extraction, PCR, and sequencing) and by an unrestricted grant from NexBio, Inc (rhinovirus challenge study and analysis of sequencing data). Ms Allen received support from the National Institute of General Medical Sciences of the National Institutes of Health under Award Number T32GM008715.

\section{Author details}

${ }^{1}$ Center for Public Health Genomics, University of Virginia, PO Box 800717, Charlottesville, USA. ${ }^{2}$ Department of Biochemistry and Molecular Genetics, University of Virginia, Charlottesville, USA. ${ }^{3}$ Bioinformatics Core Facility, University of Virginia, Charlottesville, USA. ${ }^{4}$ Department of Pediatrics, University of Virginia, Charlottesville, USA. ${ }^{5}$ Department of Otolaryngology, University of Virginia, Charlottesville, USA. ${ }^{6}$ Department of Medicine, University of Virginia, Charlottesville, USA.

Received: 1 November 2013 Accepted: 28 May 2014

Published: 25 June 2014

\section{References}

1. Chi DH, Hendley JO, French P, Arango P, Hayden FG, Winther B: Nasopharyngeal reservoir of bacterial otitis media and sinusitis pathogens in adults during wellness and viral respiratory illness. Am J Rhinol 2003, 17:209-214.

2. Hendley JO, Hayden FG, Winther B: Weekly point prevalence of Streptococcus pneumoniae, Hemophilus influenzae and Moraxella catarrhalis in the upper airways of normal young children: effect of respiratory illness and season. APMIS 2005, 113:213-220.
3. Puhakka T, Mäkelä MJ, Malmström K, Uhari M, Savolainen J, Terho EO, Pulkkinen $\mathrm{M}$, Ruuskanen O: The common cold: effects of intranasal fluticasone propionate treatment. J Allergy Clin Immunol 1998, 101:726-731.

4. Bogaert D, Keiijser B, Huse S, Rossen J, Veenhoven R, van Gils E, Bruin J, Montijn R, Bonten M, Sanders E: Variability and diversity of nasopharyngeal microbiota in children: a metagenomic analysis. PLoS One 2011, 6:e17035.

5. Ling Z, Liu X, Luo Y, Yuan L, Nelson KE, Wang Y, Xiang C, Li L: Pyrosequencing analysis of the human microbiota of healthy Chinese undergraduates. BMC Genomics 2013, 14:390.

6. Hilty M, Qi W, Brugger SD, Frei L, Agyeman P, Frey PM, Aebi S, Muhlemann K: Nasopharyngeal microbiota in infants with acute otitis media. J Infect Dis 2012, 205:1048-1055

7. Biesbroek G, Wang X, Keijser BJ, Ejjkemans RM, Trzcinski K, Rots NY, Veenhoven $R H$, Sanders EA, Bogaert D: Seven-valent pneumococcal conjugate vaccine and nasopharyngeal microbiota in healthy children. Emerg Infect Dis 2014, 20:201-210.

8. Pettigrew MM, Laufer AS, Gent JF, Kong Y, Fennie KP, Metlay JP: Upper respiratory tract microbial communities, acute otitis media pathogens, and antibiotic use in healthy and sick children. Appl Environ Microbiol 2012 78:6262-6270.

9. Wang Q, Garrity GM, Tiedje JM, Cole JR: Naive Bayesian classifier for rapid assignment of rRNA sequences into the new bacterial taxonomy. Appl Environ Microbiol 2007, 73:5261-5267.

10. van der Zalm MM, van Ewijk BE, Wilbrink B, Uiterwaal CS, Wolfs TF, van der Ent CK: Respiratory pathogens in children with and without respiratory symptoms. J Pediatr 2009, 154:396-400. 400 e391.

11. Murphy TF, Bakaletz LO, Smeesters PR: Microbial interactions in the respiratory tract. Pediatr Infect Dis J 2009, 28:S121-S126.

12. Peltola VT, McCullers JA: Respiratory viruses predisposing to bacterial infections: role of neuraminidase. Pediatr Infect Dis J 2004, 23:587-597.

13. van den Bergh MR, Biesbroek G, Rossen JW, de Steenhuijsen Piters WA, Bosch AA, van Gils EJ, Wang X, Boonacker CW, Veenhoven RH, Bruin JP: Associations between pathogens in the upper respiratory tract of young children: interplay between viruses and bacteria. PLoS One 2012, 7:e47711.

14. Brogden KA, Guthmiller JM, Taylor CE: Human polymicrobial infections. Lancet 2005, 365:253-255.

15. Avadhanula V, Rodriguez CA, Devincenzo JP, Wang Y, Webby RJ, Ulett GC, Adderson EE: Respiratory viruses augment the adhesion of bacterial pathogens to respiratory epithelium in a viral species- and cell typedependent manner. J Virol 2006, 80:1629-1636.

16. Tong HH, Weiser JN, James MA, DeMaria TF: Effect of influenza A virus infection on nasopharyngeal colonization and otitis media induced by transparent or opaque phenotype variants of Streptococcus pneumoniae in the chinchilla model. Infect Immun 2001, 69:602-606.

17. McNamee LA, Harmsen AG: Both influenza-induced neutrophil dysfunction and neutrophil-independent mechanisms contribute to increased susceptibility to a secondary Streptococcus pneumoniae infection. Infect Immun 2006, 74:6707-6721.

18. Colamussi ML, White MR, Crouch E, Hartshorn KL: Influenza A virus accelerates neutrophil apoptosis and markedly potentiates apoptotic effects of bacteria. Blood 1999, 93:2395-2403.

19. Abramson JS, Hudnor HR: Role of the sialophorin (CD43) receptor in mediating influenza A virus-induced polymorphonuclear leukocyte dysfunction. Blood 1995, 85:1615-1619.

20. Hyde ER, Petrosino JF, Piedra PA, Camargo CA Jr, Espinola JA, Mansbach JM: Nasopharyngeal Proteobacteria are associated with viral etiology and acute wheezing in children with severe bronchiolitis. J Allergy Clin Immunol 2014, 133:1220-1222.

21. Molyneaux PL, Mallia P, Cox MJ, Footitt J, Willis-Owen SA, Homola D, Trujillo-Torralbo MB, Elkin S, Kon OM, Cookson WO, Moffatt MF, Johnston S: Outgrowth of the bacterial airway microbiome after rhinovirus exacerbation of chronic obstructive pulmonary disease. Am J Respir Crit Care Med 2013, 188:1224-1231.

22. Allen EK, Pitkaranta A, Maki M, Hendley JO, Laakso S, Sale MM, Winther B: Bacteria in the nose of young adults during wellness and rhinovirus colds: detection by culture and microarray methods in 100 nasal lavage specimens. Int Forum Allergy Rhinol 2013, 3:731-739.

23. Hamparian W: Rhinoviruses. In Diagnostic procedures for viral, rickettsial and chlamydial infections. Edited by Lennette EH, Schmidt NJ. Washington, D.C: American Public Health Association; 1979:562. 
24. Ren T, Glatt DU, Nguyen TN, Allen EK, Early SV, Sale M, Winther B, Wu M: $16 \mathrm{~S}$ rRNA survey revealed complex bacterial communities and evidence of bacterial interference on human adenoids. Environ Microbiol 2013, 15:535-547.

25. FastQC A quality control tool for high throughput sequence data. http://www.bioinformatics.babraham.ac.uk/projects/fastqc/.

26. Edgar RC: Search and clustering orders of magnitude faster than BLAST. Bioinformatics 2010, 26:2460-2461.

27. Caporaso JG, Kuczynski J, Stombaugh J, Bittinger K, Bushman FD, Costello EK, Fierer N, Pena AG, Goodrich JK, Gordon Jl, Huttley GA, Kelley ST, Knights D, Koenig JE, Ley RE, Lozupone CA, McDonald D, Muegge BD, Pirrung M, Reeder J, Sevinsky JR, Turnbaugh PJ, Walters WA, Widmann J, Yatsunenko T, Zanveld J, Knight R: QIIME allows analysis of high-throughput community sequencing data. Nat Methods 2010, 7:335-336.

28. DeSantis TZ, Hugenholtz P, Larsen N, Rojas M, Brodie EL, Keller K, Huber T, Dalevi D, Hu P, Andersen GL: Greengenes, a chimera-checked 16S rRNA gene database and workbench compatible with ARB. Appl Environ Microbiol 2006, 72:5069-5072

29. Edgar RC, Haas BJ, Clemente JC, Quince C, Knight R: UCHIME improves sensitivity and speed of chimera detection. Bioinformatics 2011, 27:2194-2200.

30. Caporaso JG, Bittinger K, Bushman FD, DeSantis TZ, Andersen GL, Knight R: PyNAST: a flexible tool for aligning sequences to a template alignment. Bioinformatics 2010, 26:266-267.

31. Price MN, Dehal PS, Arkin AP: FastTree 2-approximately maximumlikelihood trees for large alignments. PLoS One 2010, 5:e9490.

32. Lozupone C, Knight R: UniFrac: a new phylogenetic method for comparing microbial communities. Appl Environ Microbiol 2005, 71:8228-8235.

33. Ramette A: Multivariate analyses in microbial ecology. FEMS Microbiol Ecol 2007, 62:142-160.

34. Costello EK, Lauber CL, Hamady M, Fierer N, Gordon Jl, Knight R: Bacterial community variation in human body habitats across space and time. Science 2009, 326:1694-1697.

35. Biesbroek G, Sanders EA, Roeselers G, Wang X, Caspers MP, Trzcinski K, Bogaert D, Keijser BJ: Deep sequencing analyses of low density microbial communities: working at the boundary of accurate microbiota detection. PLoS One 2012, 7:e32942.

36. Segata N, Waldron L, Ballarini A, Narasimhan V, Jousson O, Huttenhower C: Metagenomic microbial community profiling using unique clade-specific marker genes. Nat Methods 2012, 9:811-814.

37. Murphy TF, Brauer AL, Sethi S, Kilian M, Cai X, Lesse AJ: Haemophilus haemolyticus: a human respiratory tract commensal to be distinguished from Haemophilus influenzae. J Infect Dis 2007, 195:81-89.

38. McCrea KW, Xie J, LaCross N, Patel M, Mukundan D, Murphy TF, Marrs CF, Gilsdorf JR: Relationships of nontypeable Haemophilus influenzae strains to hemolytic and nonhemolytic Haemophilus haemolyticus strains. J Clin Microbiol 2008, 46:406-416.

39. Gwaltney JM, Hendley JO, Phillips CD, Bass CR, Mygind N, Winther B: Nose blowing propels nasal fluid into the Paranasal Sinuses. Clin Infect Dis 2000, 30:387-391.

40. Schwartz RH, Pitkaranta A: Computed tomography imaging of the Maxillary and Ethmoid Sinuses in children with short-duration purulent Rhinorrhea. Otolaryngol Head Neck Surg 2001, 124:160-163.

41. Winther B, Gwaltney JM, Phillips CD, Hendley JO: Radiopaque contrast dye in nasopharynx reaches the middle ear during swallowing and/or yawning. Acta Otolaryngol 2005, 125:625-628.

42. Segata N, Boernigen D, Tickle TL, Morgan XC, Garrett WS, Huttenhower C: Computational meta'omics for microbial community studies. Mol Syst Biol 2013, 9:666.

43. Willner D, Furlan M, Haynes M, Schmieder R, Angly FE, Silva J, Tammadoni S, Nosrat B, Conrad D, Rohwer F: Metagenomic analysis of respiratory tract DNA viral communities in cystic fibrosis and non-cystic fibrosis individuals. PLoS One 2009, 4:e7370.

doi:10.1186/2049-2618-2-22

Cite this article as: Allen et al:: Characterization of the nasopharyngeal microbiota in health and during rhinovirus challenge. Microbiome 2014 2:22.

\section{Submit your next manuscript to BioMed Central and take full advantage of:}

- Convenient online submission

- Thorough peer review

- No space constraints or color figure charges

- Immediate publication on acceptance

- Inclusion in PubMed, CAS, Scopus and Google Scholar

- Research which is freely available for redistribution
C Biomed Central 